\title{
Atomistic modeling study of surface effect on oxide ion diffusion in yttria-stabilized zirconia
}

\author{
Albert M. Iskandarov ${ }^{\mathrm{a}, \mathrm{b}, \mathrm{c}, *}$, Yoshitaka Umeno ${ }^{\mathrm{a}, \mathrm{b}}$ \\ ${ }^{a}$ Institute of Industrial Science, The University of Tokyo, 4-6-1 Komaba, Meguro-ku, \\ Tokyo 153-8505, Japan \\ ${ }^{b}$ CREST, Japan Science and Technology Agency, K's Gobancho, 7, Chiyoda-ku, Tokyo \\ 102-0076, Japan \\ ${ }^{c}$ Institute for Metals Superplasticity Problems, Russian Academy of Sciences, Khalturin \\ St. 39, Ufa 450001, Russia
}

\begin{abstract}
This paper reports results of molecular dynamics (MD) study of oxide ion diffusion in cubic ytrria-stabilized zirconia (YSZ) using our recently developed interatomic potential [A.M. Iskandarov et al.; J. Phys.: Condens. Matter 27, 015005 (2015)], which is based on the Tangney-Scandolo dipole model. We demonstrate that our potential can reproduce oxide ion conductivity in bulk YSZ with yttria concentration varying from 2 to $14 \mathrm{~mol} \%$. We confirm significant effect of free surfaces on oxygen diffusion by direct MD calculations at elevated temperatures and nudged elastic band analyses. It was found that oxygen vacancies tend to migrate from the (111) surface and locate in the bulk region, suppressing local oxide ion diffusion at the surface. In contrast, higher oxygen vacancy concentration and activation of oxide ion jumps along $\langle 110\rangle$ direction facilitate oxygen diffusion at the (110) surface. The MD results for the (110) surface are supported by density functional theory calculations.
\end{abstract}

Keywords: yttria-stabilized zirconia, oxide ion diffusion, surface, atomistic simulation

PACS: 68.35.Fx, 31.15.xv

\footnotetext{
${ }^{*}$ Corresponding author

Email address: albert@ulab.iis.u-tokyo.ac.jp (Albert M. Iskandarov)
} 


\section{Introduction}

Ionic conductivity is a crucial property for the materials used as an electrolyte in solid oxide fuel cells (SOFCs). Thus, it is the cubic yttria-stabilized zirconia (YSZ) that is commonly used as an electrolyte and anode component in SOFC. Indeed, compared with a pure zirconia, YSZ possesses high ionic conductivity arising from a high density of oxygen vacancies that are generated to meet charge neutrality condition after yttria doping, when two $\mathrm{ZrO}_{2}$ units are replaced by one $\mathrm{Y}_{2} \mathrm{O}_{3}$ unit.

There have been many theoretical and experimental studies to understand mechanisms of oxide ion diffusion in YSZ $[1,2,3,4,5,6,7,8,9]$. Kondoh et al. [1] experimentally studied the effect of aging and yttria concentration on ionic conductivity of YSZ. Before aging, the lowest activation energy $(0.7 \mathrm{eV})$ of oxide ion migration was obtained for $8 \mathrm{~mol} \%$ YSZ. With aging, conductivity decreased, which was accompanied with the increase of the activation energy. Though the number of oxygen vacancies constantly increases when yttria concentration increases, conductivity starts to decrease for yttria concentrations above $8 \mathrm{~mol} \%$. This effect can be explained by the fact that the concentration of yttrium dopants also increases, and that the oxide ions migrate near them with a smaller rate. Indeed, by molecular dynamics (MD) simulations, Kilo et al.[10] analyzed relative frequency of oxide ion jumps between different combinations of adjacent cations. The results indicate that oxide ions are more likely to migrate between two $\mathrm{Zr}$ ions than between Zr and Y, or even more so between two Y ions. This explanation agrees with other simulation results based on empirical interatomic potentials [6, 11]. More recently, Pornprasertsuk et al. [12] performed density functional theory (DFT) study of the energy barriers needed for an oxide ion to migrate to its vacant neighbor positions. It was confirmed that the barriers depend strongly on local atomic environment around the migrating ion. For instance, the barrier is $0.67 \mathrm{eV}$ when two tetrahedra containing the migrating oxide ion and the oxygen vacancy are formed by six $\mathrm{Zr}$ ions, while it is $1.23 \mathrm{eV}$ when the oxide ion migrates between two $\mathrm{Y}$ ions with four others being Zr. Krishnamurthy et al. [13] estimated these two migration energies equal to $0.58 \mathrm{eV}$ and $1.86 \mathrm{eV}$, respectively. Due to the lower energy barriers between two $\mathrm{Zr}$ cations, it is theoretically possible to arrange $\mathrm{Y}$ dopants in such a way that they provide channels of enhanced oxide ion diffusion. For instance, Okazaki et al. considered bulk $20 \mathrm{~mol} \%$ YSZ where Y dopants form (100) metallic layers. This layered structure was reported to possess a higher 
diffusion coefficient compared with random distribution of $\mathrm{Y}$ ions $[14,15]$. Enhanced oxide ion conductivity was also recently reported by Lee et al. for YSZ structures with Y dopants aggregated in [100] lines [16].

Local environment and the migration barriers, depend also on applied strain. Kushima et al. [8] studied the effect of biaxial tensile strain on the process of oxide ion migration. It was revealed that relatively small biaxial tensile strain causes weakening of the bonds between the migrating oxide ion and the nearest cations, and that the strain also increases space for the oxide ion between the cations. This effect results in the strikingly low migration energy equal to $0.1 \mathrm{eV}$ at $4 \%$ strain and consecutive significant enhancement of oxygen diffusivity by several orders of magnitude. However, further increase of the applied strain leads to breaking of one bond and strengthening of other bonds between the oxide ion and the closest cations. This increases the energy needed to break these stronger bonds and increases the migration barrier. Suzuki et al. [17] conducted a MD study of oxygen diffusion of YSZ component in YSZ/ $\mathrm{CeO}_{2}$ superlattice reported a 1.7 times higher diffusion coefficient compared with that in bulk YSZ, which was also attributed to a strained state of YSZ due to larger lattice parameter of ceria.

The effect of compressive and dilatative strain can also explain ionic conductivity of heterostructures forming coherent interfaces [18, 19, 20, 21]. Small lattice mismatch between two adjacent layers at a coherent interface can be fully compensated by elastic strains. For instance, Korte et al. [22] analyzed conductivity of a YSZ $/ \mathrm{Y}_{2} \mathrm{O}_{3}$ multilayer stack. The layers were epitaxially (111)-oriented. Their thickness was varied while the total height of the stack was kept fixed. The increase of conductivity by a factor of 1.4 was observed when the number of interfaces was increased to 20. Having not observed periodic alignment of misfit dislocations, which are likely to form at semicoherent interfaces, the authors concluded that the $3 \%$ lattice misfit was compensated by elastic expansion of the YSZ interfacial region.

Crystal symmetry is broken and local atomic environment of ionic solids is changed not only at interfaces of heterostructures but also at free surfaces. Moreover, the equilibrium structure of YSZ surfaces can be notably different from that of ideal cut of bulk. For instance, DFT and MD calculations show that while the structure of the oxygen (O-) terminated (111) surface of cubic zirconia undergoes only minor relaxation, displacements of atoms at the (110) zirconia surface can reach $0.2 \AA[23,24,25,26]$. The structure of a doped (110) zirconia surface is even more complicated. Indeed, oxide ions that are nearest neighbors (NN) to oxygen vacancies migrate towards 
them by a half of initial distance between them, raising over the surface by around $0.7 \AA[26,25]$. Such significant change of atomic structures at free YSZ surfaces can significantly influence energy barriers for oxide ion migration, which, in turn, can result in a change of subsurface diffusion and affect reaction rates [27]. Importance of oxygen diffusion was also emphasized by Ammal et al. by combined DFT and microkinetic study of various reaction pathways of hydrogen oxidation at TPB formed at Ni(111)/YSZ(111) interface [28]. The results indicate that oxygen diffusion in YSZ is a rate limiting process in low temperature conditions $(<1000 \mathrm{~K})$.

In the present paper we first employ MD calculations to demonstrate that our interatomic potential can successfully describe oxygen diffusion in bulk YSZ with yttria concentration of 2-14 mol \%. The ability of the potential to accurately reproduce energies and structures of YSZ surfaces [25] allowed us to further address a problem of oxide ion diffusion near the (110) and O-terminated (111) surfaces. In spite of the abundance of DFT studies regarding the (111) YSZ surfaces, there are only few considering the (110) surface. Therefore, we performed additional DFT calculations to support our main findings for the (110) surface.

This paper is organized as follows. In Sec. 2 we describe the dipole model and other simulation details. In Sec. 3.1 we present results for oxide ion diffusion in bulk YSZ. MD results of oxygen diffusion near the (111) surface is discussed in Sec. 3.2.1-3.2.2, near the (110) - in Sec. 3.2.3-3.2.4. Section 3.2.5 addresses validation of the main MD results for the (110) surface by DFT calculations.

\section{Computational setup}

\subsection{Dipole model}

According to the dipole model proposed by Tangney and Scandolo (TS) [29], the total potential energy $E_{\text {tot }}$ of a condensed ionic system is calculated as a sum of two contributions: the energy of a short-range pairwise interactions between ions, $E_{\text {tot }}^{\mathrm{sr}}$, and the energy of a long-range electrostatic interactions, $E_{\text {tot }}^{\mathrm{el}}$. The short-range interactions are described by the Buckingham potential, which for a pair of atoms $i$ and $j$ can be written as

$$
E_{i j}^{\mathrm{sr}}\left(r_{i j}\right)=a_{i j} e^{-r_{i j} / b_{i j}}-c_{i j}\left(\frac{b_{i j}}{r_{i j}}\right)^{6},
$$


where $r_{i j}$ is the distance between the atoms, and $a_{i j}, b_{i j}$, and $c_{i j}$ are the parameters of the Buckingham potential, which depend only on types of atoms $i$ and $j$. The value of $E_{\text {tot }}^{\mathrm{sr}}$ is calculated as a sum of $E_{i j}^{\mathrm{sr}}\left(r_{i j}\right)$ for all pairs of atoms $i$ and $j$ located within a cut-off radius $R_{\mathrm{c}}^{\mathrm{sr}}=10 \AA$ i.e.,

$$
E_{\mathrm{tot}}^{\mathrm{sr}}=\sum_{i=1}^{N} \sum_{j>i, r_{i j} \leq R_{\mathrm{c}}^{\mathrm{sr}}}^{N} E_{i j}^{\mathrm{sr}}
$$

where $N$ is the number of atoms in the system.

A distinctive feature of the TS model is the incorporation of electrostatic dipole moments $\left\{\mathbf{p}_{i}\right\}$ induced on ions. This allows accounting for charge polarization of the ions. Thus, the total energy of electrostatic interactions, $E_{\text {tot }}^{\mathrm{el}}$, consists of the energy of electrostatic interactions, $E^{q q}$, between ions' point charges $\left\{q_{j}\right\}$, and two more energy terms, namely

$$
E_{\mathrm{tot}}^{\mathrm{el}}=E^{q q}+E^{p q}+E^{p p}
$$

where $E^{p q}$ represents electrostatic interactions between point charges and dipoles, and $E^{p p}$ between dipoles.

To calculate the latter two terms in equation (3), one has to determine the dipole moments $\left\{\mathbf{p}_{i}\right\}$, which are formed by two contributions. The first contribution to $\mathbf{p}_{i}$ comes from the point charges $\left\{q_{j}\right\}$ of surrounding ions within a cut-off radius of $R_{\mathrm{c}}^{\mathrm{el}}=14 \AA$, and can be written as

$$
\mathbf{p}_{i}^{\mathrm{P}}=\alpha_{i} \sum_{j=1, j \neq i, r_{i j}<R_{\mathrm{c}}^{\mathrm{el}}}^{j=N} \frac{q_{j} \mathbf{r}_{i j}}{r_{i j}^{3}} f_{i j}\left(\mathbf{r}_{i j}\right),
$$

where

$$
f_{i j}\left(r_{i j}\right)=\gamma_{i j} \sum_{k=0}^{4} \frac{\left(\beta_{i j} r_{i j}\right)^{k}}{k !} e^{-\beta_{i j} r_{i j}} .
$$

$\alpha_{i}$ is the polarizability of the $i$-th ion, $\beta_{i j}$ and $\gamma_{i j}$ are parameters of the model [30]. Since the TS model does not take into account charge transfer effect, the value of $\mathrm{p}^{\mathrm{P}}$ in equation (4) is determined by the positions of surrounding ions. The second contribution to $\mathbf{p}_{i}$ comes from electric field $\mathbf{E}\left(\mathbf{r}_{i}\right)$ imposed by the dipole moments $\left\{\mathbf{p}_{j}\right\}$ of surrounding ions with the same cut-off radius $R_{\mathrm{c}}^{\mathrm{el}}$. Thus, total induced dipole moments $\left\{\mathbf{p}_{i}\right\}$ can be determined only in a self-consistent iterative manner. Specifically, if at the 
present iterative step the total dipole moments are equal to $\left\{\mathbf{p}_{i}^{n}\right\}$, then the next iteration gives

$$
\mathbf{p}_{i}^{n+1}=\alpha_{i} \mathbf{E}\left(r_{i},\left(\mathbf{p}_{j}^{n}, \mathbf{r}_{j}^{n}\right)\right)+\mathbf{p}_{i}^{\mathrm{P}} .
$$

Having calculated the dipole moments, one can calculate the total electrostatic energy $E_{\text {tot }}^{\mathrm{el}}$ using the following expressions for the terms in the right hand side of equation (3):

$$
\begin{array}{r}
E^{\mathrm{qq}}=\frac{1}{2} \sum_{i=1}^{N} \sum_{j=1, j \neq i}^{N} \frac{q_{i} q_{j}}{r_{i j}}, \\
E^{\mathrm{pq}}=\frac{1}{2} \sum_{i=1}^{N} \sum_{j=1, j \neq i}^{N} \frac{1}{r_{i j}^{3}}\left[q_{i}\left(\mathbf{p}_{j} \cdot \mathbf{r}_{i j}\right)\right. \\
\left.-q_{j}\left(\mathbf{p}_{i} \cdot \mathbf{r}_{i j}\right)\right], \\
E^{\mathrm{pp}}=\frac{1}{2} \sum_{i=1}^{N}\left[\frac{p_{i}^{2}}{\alpha_{i}}+\sum_{j=1, j \neq i}^{N} \frac{1}{r_{i j}^{3}}\left[\mathbf{p}_{i} \cdot \mathbf{p}_{j}\right.\right. \\
\left.\left.-\frac{3}{r_{i j}^{2}}\left(\mathbf{p}_{i} \cdot \mathbf{r}_{i j}\right)\left(\mathbf{p}_{j} \cdot \mathbf{r}_{i j}\right)\right]\right] .
\end{array}
$$

We used a method proposed by Wolf et al. [31] to perform summation of these three contributions to the total electrostatic energy. Discussion of the parameter selection for the summation can be found elsewhere [32, 33]. In the present paper all electrostatic energies were calculated with the same cut-off radius $R_{\mathrm{c}}^{\mathrm{el}}=14 \AA$.

The TS model for YSZ has 35 independent parameters to be determined. We performed parametrization of the model based on DFT reference data for bulk and slab YSZ systems. The derived parameters and details of the parametrization can be found in our previous paper [25].

\subsection{Oxide ion diffusion in bulk $Y S Z$}

To estimate oxide ion conductivity in bulk YSZ, we performed MD simulations at temperatures from $1000 \mathrm{~K}$ to $1750 \mathrm{~K}$ and for five dopant concentrations, i.e. 2, 6, 8, 10 and $14 \mathrm{~mol} \% \mathrm{Y}_{2} \mathrm{O}_{3}$. Simulations were performed for NPT ensemble under vanishing stress components that were controlled by the Parinello-Rahman method [34]. The temperature was kept constant using velocity scaling at each time step. The mean square displacement $\left\langle r^{2}(t)\right\rangle$ 
(MSD) was calculated at every time step, $t$, over all oxide ions as

$$
\left\langle r^{2}(t)\right\rangle=\frac{\sum_{i=1}^{N}\left[\mathbf{r}_{i}(t)-\mathbf{r}_{i}(0)\right]^{2}}{N},
$$

where $r_{i}(t)$ is the position of the $i$-th oxide ion at time $t$, and $N$ is the total number of oxide ions in the system. The diffusion coefficient of oxide ions, $D_{\mathrm{O}}$, can be further evaluated from a sufficiently long simulation as

$$
D_{\mathrm{O}}=\lim _{t \rightarrow \infty} \frac{\left\langle r^{2}(t)\right\rangle}{6 t} .
$$

Using $D_{\mathrm{O}}$ one can calculate the conductivity of oxide ions, $\sigma_{\mathrm{O}}$, as

$$
\sigma_{\mathrm{O}}=\frac{N_{\mathrm{O}} q_{\mathrm{O}} D_{\mathrm{O}}}{k_{\mathrm{B}} T}
$$

where $k_{\mathrm{B}}$ is the Boltzmann constant, $T$ the temperature in simulation, and $N_{\mathrm{O}}$ the density of oxide ions.

The activation energy of oxide ion diffusion, $\Delta E_{\text {act }}$, for each yttria concentration can be extracted from fitting temperature dependence of $D_{\mathrm{O}}(T)$ to the Arrhenius relationship

$$
D_{\mathrm{O}}=D_{\mathrm{O}}^{0} \exp \left(-\frac{\Delta E_{\mathrm{act}}}{k_{B} T}\right),
$$

where $D_{\mathrm{O}}^{0}$ is the pre-exponential diffusion constant.

\subsection{Oxide ion diffusion near YSZ surfaces}

To study oxygen diffusion near free surfaces, we considered YSZ slab models with different distributions of yttrium dopants. In order to quantitatively evaluate the distribution of oxygen vacancies, we divided the slabs into imaginary layers of equal thickness. The layers are parallel to the surfaces and shown schematically in Fig. 1 by the vertical dashed lines. We calculated the number of oxygen vacancies and yttrium dopants within each layer at each time step. Moreover, to estimate the activity of oxygen migrations within each layer, we calculated the distribution of in-plane MSD over the layers as follows. Contribution of an atom entering $i$-th layer at the point $\mathrm{B}$ to the layer's in-plane MSD is calculated as squared projection $\mathbf{r}_{x y}^{i}$ of the atoms's displacement $\mathbf{r}^{i}$ within the layer on the surface plane. Every time the atom 


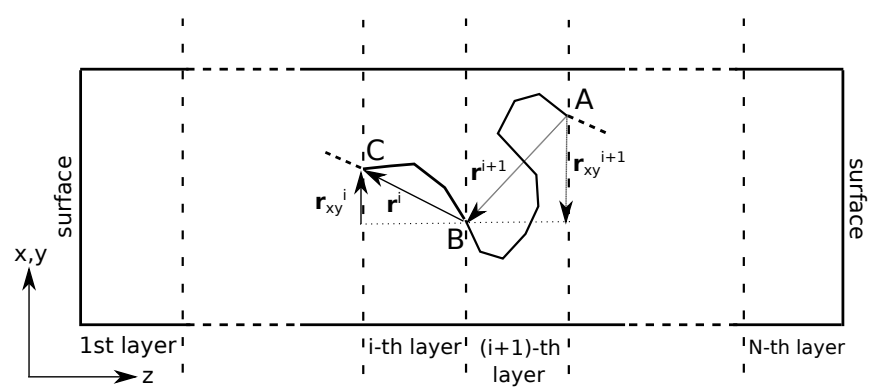

Figure 1: Schematic illustration of MSD distribution calculation. Contribution of an atom's migration along $\mathrm{A}-\mathrm{B}-\mathrm{C}$ path to in-plane MSD of the $i$-th layer is equal to the squared projection $\mathbf{r}_{x y}^{i}$ of its displacement $\mathbf{r}^{i}$ within the layer on $x O y$ plane.

repeatedly enters the layer, its displacement is independently added to the MSD.

Since we are mainly interested in oxygen diffusion, we did not try to evaluate equilibrium distribution of yttrium dopants but focused on a general features of oxygen distribution for two types of distribution of the dopants: even distribution along the direction perpendicular to the surface and random one. Results of the MD calculations at elevated temperatures are presented from analysis of a steady-state when neither oxygen vacancy distribution nor MSD distribution changed, being averaged over sufficiently long period of time.

\subsection{DFT setup}

Density functional calculations were performed by the plane wave code VASP [35, 36]. We used projector-augmented wave method [37] and the Perdew-Burke-Ernzerhof exchange-correlation functional, which is based on the generalized gradient approximation. Energy of plane waves was limited by the cut-off energy of $500 \mathrm{eV}$ according to Xia [38]. Brillouin zone was sampled using $4 \times 4 \times 4$ and $2 \times 2 \times 1$ Monkhorst-Pack $k$-point meshes for bulk and slab structures, respectively. A vacuum layer of $10 \AA$ was introduced in the slab systems to avoid interactions with periodic images of the surfaces. Further details regarding geometry and composition of the examined structures are provided individually in the corresponding sections. All structural relaxations were performed with fixed simulation cell size until all force components were less than $0.01 \mathrm{eV} / \AA$. 


\section{Results and discussion}

\subsection{Oxide ion conductivity in bulk YSZ}

In this section we firstly study energy barriers for elementary jumps of three oxide ions in bulk YSZ towards position of the oxygen vacancy $\left(\mathrm{V}_{\mathrm{o}}\right)$ shown in the inset image in Fig. 2. All three examined paths are along $\langle 100\rangle$ directions. The studied structure contained 95 atoms including two yttrium dopants and the oxygen vacancy, which corresponds to $3.2 \mathrm{~mol} \% \mathrm{Y}_{2} \mathrm{O}_{3}$. We calculated energies of some intermediate structures along these paths by DFT and our interatomic potential. For these calculations we performed relaxation of neither edge structures, nor intermediate ones. Therefore, all cations and oxide ions were positioned in the sites of ideal fluorite structure. This allows us to compare MD and DFT energy profiles derived for the same structures, avoiding possible differences due to relaxation of the edge and intermediate structures, which is inevitable in a nudged elastic band (NEB) analysis. Results are presented in Fig. 2 in terms of relative energy of system due to the migration of the oxide ions. Our potential reproduces with reasonable accuracy not only the positions of the local minimums and maximums along the paths but also the height of the energy barriers that oxide ion has to overcome during migration; for all sampled points the difference with DFT data is less than $0.2 \mathrm{eV}$. The edge configurations do not form local minimums along all three paths, the minimums are rather located around positions 0.2 and 0.8. This indicates that the oxide ions relax by shift towards the vacancy from their sites in the ideal fluorite structure. Moreover, the deepest minimum is located at position 0.8 along path $\mathrm{A}$, which indicates that the vacancy gets trapped near the dopants, and it has to overcome larger energy barrier to return to the initial position along this path than along the other two. This supports the conjecture of suppressed oxygen diffusion near yttrium dopants.

In Fig. 3 we present the oxide ion conductivity $\sigma_{\mathrm{O}}$ calculated according to equation (12). We examined five yttria concentrations: 2, 6, 8, 10, and $14 \mathrm{~mol} \%$, and four temperatures: 1000, 1250, 1500, and $1750 \mathrm{~K}$. The conductivity varies from 0.02 to $0.16 \mathrm{~S} \mathrm{~cm}^{-1}$ for the considered range of parameters. The obtained conductivity range is consistent with numerous experimental results $[39,1,40,41]$ and simulations [10, 4, 42]. For instance, Kondoh et al. experimentally obtained conductivity range for $8 \mathrm{~mol} \% \mathrm{YSZ}$ around $0.025-0.55 \mathrm{~S} \mathrm{~cm}^{-1}$ in the temperature interval from 1073 to $1573 \mathrm{~K}$ [1]. For the same dopant concentration, the estimation by Lau et al. for tempera- 


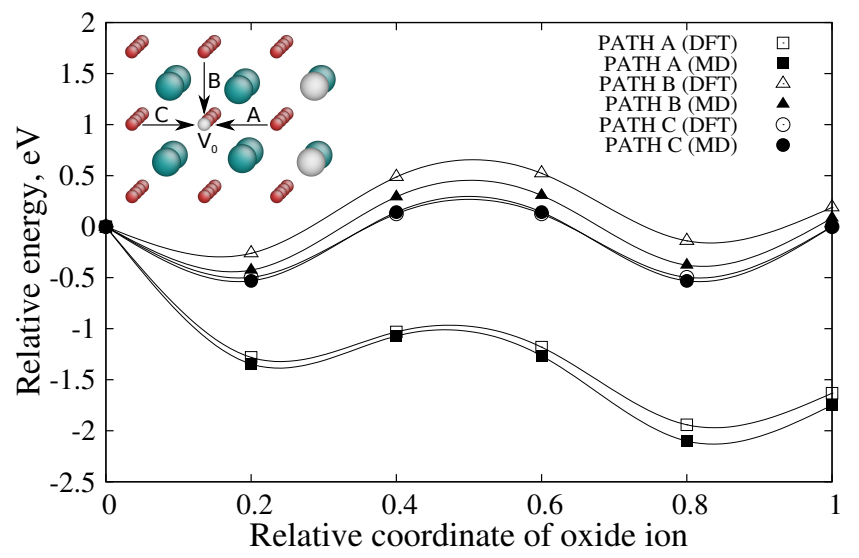

Figure 2: Energy profiles for migration of oxide ions to the initial position $\mathrm{V}_{0}$ of the oxygen vacancy along $\mathrm{A}, \mathrm{B}$, and $\mathrm{C}$ paths shown in the inset image. Filled symbols stand for MD results, open - DFT. In the inset image big blue atoms correspond to zirconium ions, big white - yttrium ions, small red - oxide ions, small white - oxygen vacancy. Arrows indicate directions of oxide ions' migration.

tures between 800 and $1200 \mathrm{~K}$ is around $0.005-0.07 \mathrm{~S} \mathrm{~cm}^{-1}$, [42] which was obtained by MD simulations based on the shell model [43].

Optimal yttria concentration in terms of maximal conductivity of oxide ions is equal to $8 \mathrm{~mol} \%$ at all sampled temperatures, which also agrees with experimental and theoretical data $[1,42]$. For $8 \mathrm{~mol} \% \mathrm{YSZ}$ we obtained $\Delta E_{\text {act }}=0.47 \mathrm{eV}$, derived from equation (13). This value is slightly below the experimentally obtained values, $0.70-1.2 \mathrm{eV}[1,40,44,45,46]$, but within the range of $0.37-1.0 \mathrm{eV}$ from previous theoretical studies $[42,6,3,10,12]$ for 8-10 mol \% YSZ. For the other four yttria concentrations, the activation energy is predictably higher than $0.47 \mathrm{eV}$ and is in the range of $0.47-0.53 \mathrm{eV}$.

\subsection{Oxide ion diffusion near YSZ surfaces}

In the previous section we demonstrated that our potential can correctly reproduce main properties of oxide ion diffusion in bulk YSZ. Moreover, in our previous work we showed that the interatomic potential used in this work can reproduce the energies and structures of clean and doped (111) and (110) zirconia surfaces [25]. Thus, we continue our study with analysis of oxide ion diffusion near two surfaces with the lowest energies, namely (111) and (110). All MD simulations in this section are performed at $1000 \mathrm{~K}$. 


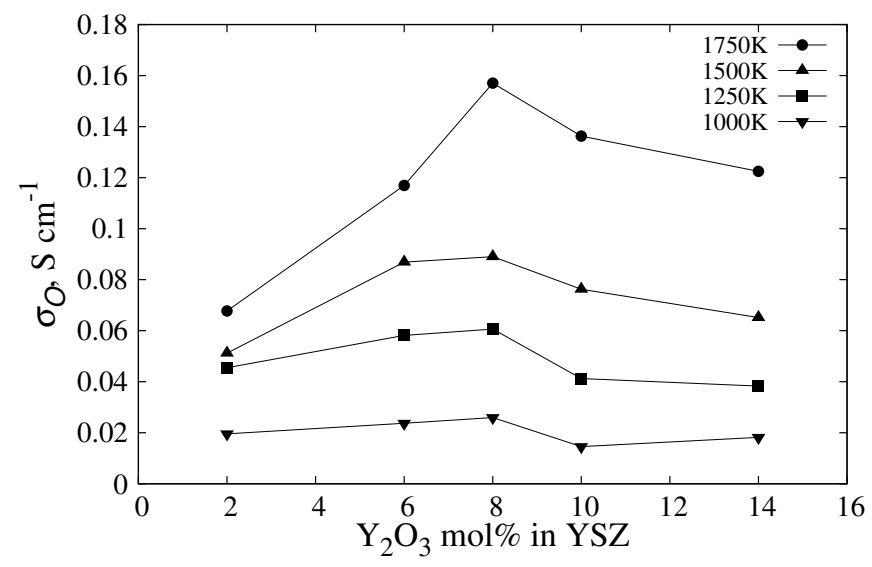

Figure 3: Oxide ion conductivity in bulk 2, 8 and $16 \mathrm{~mol} \% \mathrm{YSZ}$ at $(\mathbf{\nabla}) 1000$, 1250 $(\boldsymbol{\Lambda}) 1500$, and $(\bullet) 1750 \mathrm{~K}$.

\subsection{1. (111) surface: $M D$ at elevated temperature}

The structure of the O-terminated (111) zirconia surface can be represented as a piled-up stack of $\mathrm{O}-\mathrm{Zr}-\mathrm{O}$ trilayers. A slab configuration containing 21 of these trilayers was used for molecular dynamics simulations at $1000 \mathrm{~K}$. The dimensions of the simulation cell along $x$ and $y$ directions were equal to $18.4 \AA$ and $19.1 \AA$, respectively. The thickness of the imaginary layers for building distribution of defects and in-plane MSD was chosen to be equal to $9.1 \AA$ to hold three adjacent atomic trilayers (one trilayer triplet). This simulation cell size was confirmed to be sufficient, as no qualitative difference in results has been observed with two times larger $x$ and $y$ dimensions.

In Fig. 4 we present results for one representative case for a random distribution of yttrium dopants in the $7.9 \mathrm{~mol} \%$ YSZ slab. We show the distributions of the oxygen vacancies, in-plane MSD, and yttrium ions. The in-plane MSD and vacancy distribution were averaged over a time interval of $150 \mathrm{ps}$ at a steady state. This time span is too small to observe diffusion of yttrium ions. Thus, all yttirum ions only oscillated near their initial positions and their distribution did not change during the simulation. We can see that in the bulk region (triplets 2-6) there is a correlation between the number of $\mathrm{V}_{\mathrm{o}}$ and $\mathrm{Y}$ atoms. Indeed, one may notice that within each triplet the number of $\mathrm{V}_{\mathrm{o}}$ is approximately half that of yttrium ions, which corresponds to their stoichometric ratio. Situation is different in the surface triplets. 


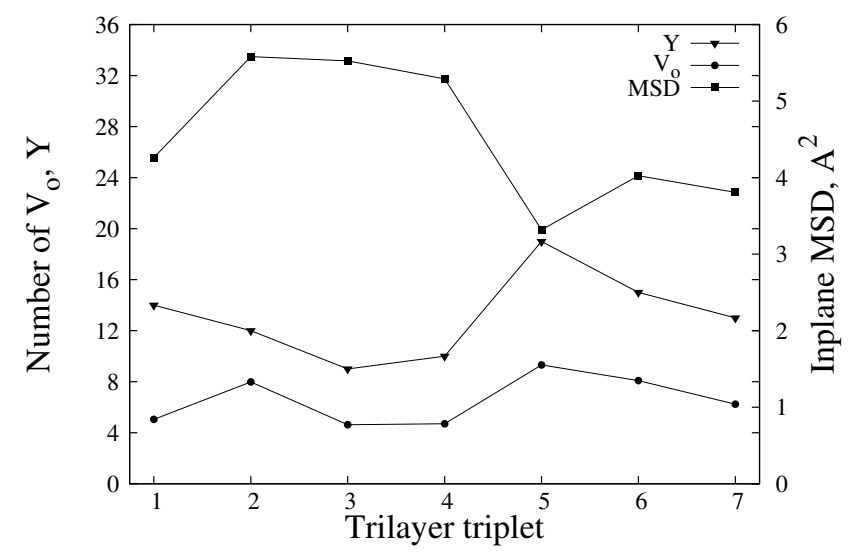

Figure 4: Distribution of yttrium dopants, oxygen vacancies and inplane MSD over trilayer triplets in $7.9 \mathrm{~mol} \%$ (111)YSZ slab. Yttrium dopants were distributed randomly.

Indeed, the first three triplets form surface with Y segregation, which was reported by Xia et al. [24] and in our previous work [25]. However, a local minimum of $V_{o}$ concentration is observed there in spite of higher yttrium concentration. Similar minimum is observed at the opposite surface, though there is a depletion of yttirum. This implies that oxygen vacancies tend to move into the bulk region rather than to stay near the surface, which also leads to the local minimums in the MSD curve.

Interestingly, according to the dopant concentration, there should be on average 6.6 oxygen vacancies and 13.2 yttrium dopants in each triplet. Triplets 2-4 contain the number of $\mathrm{V}_{\mathrm{o}}$ and yttrium dopants close to optimal concentration. Thus, it is predictably accompanied with maximum of MSD. In contrast, triplet 5 contains $19 \mathrm{Y}$ ions, which is $50 \%$ more than in even distribution. Presence of the excessive $\mathrm{Y}$ ions results in the minimum of the inplane MSD in that triplet, which supports the speculation that migration rate of the oxide ions is lower near Y ions. Qualitatively similar results were obtained for other random yttrium distributions.

Even from the presented simulation we can conclude that oxygen vacancies tend to aggregate and migrate near yttrium dopants; high local yttrium concentration suppresses oxygen migration in that region. This result agrees with negative values of $\mathrm{Y}-\mathrm{V}_{\mathrm{o}}$ binding energy at the next nearest neighbor 


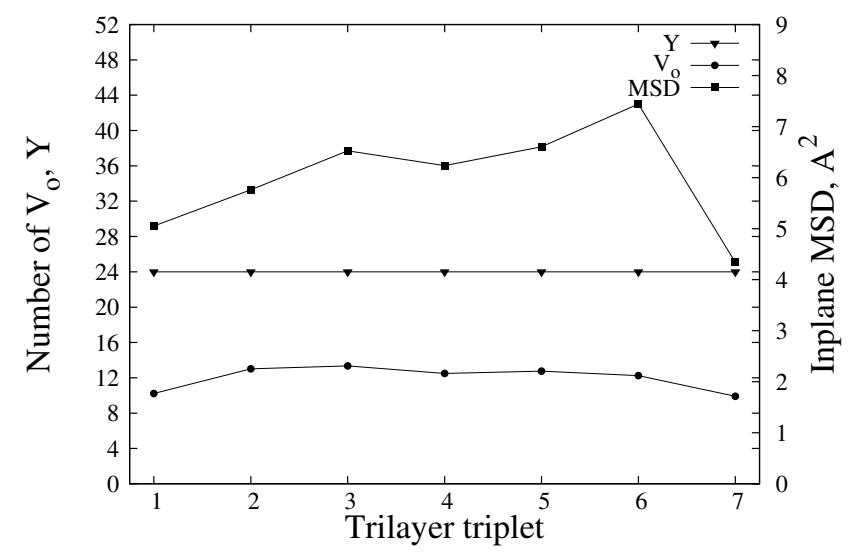

Figure 5: Distribution of yttrium dopants, oxygen vacancies and inplane MSD over trilayer triplets in $8 \mathrm{~mol} \%$ (111) YSZ slab with evenly distributed Y atoms along the slab's thickness direction.

distance (NNN) $[12,25,47]$ and increased energy barriers for oxygen migration between two $\mathrm{Y}$ ions [12]. However, to eliminate the effect of uneven yttrium distribution on oxide ion diffusion near the (111) surface, we performed similar simulation with even distribution of $\mathrm{Y}$ over O-Zr-O trilayers.

In Fig. 5 we show data similar to that in Fig. 4 but for even distribution of $\mathrm{Y}$ atoms along the thickness direction. The size of the slab was increased to $21.7 \AA$ and $25.1 \AA$ to get integer numbers of $\mathrm{Y}$ atoms (24) and oxygen vacancies (12) in each trilayer at the dopant concentration around $8 \mathrm{~mol} \%$. The slab's thickness was kept the same and contained 7 trilayer triplets. In the surface region we can see the same tendency of a lower $V_{o}$ concentration and MSD, while we see even distribution of $\mathrm{V}_{\mathrm{o}}$ in the bulk region. In order to explain tendency of $V_{o}$ migration into the bulk region we performed NEB analysis of $\mathrm{V}_{\mathrm{o}}$ migration near the surface region of the (111) YSZ slab.

\subsection{2. (111) surface: $N E B$ analysis}

We studied energy barriers for oxygen vacancy migration along three paths near the (111) surface shown in Fig. 6. Two yttirum ions are nearest neighbors to each other and are located in the outermost metallic layer to mimic yttrium segregation at the surface [24]. For paths A and B, the oxygen vacancy starts migration from $\mathrm{NN}$ position to the dopants. While moving along path A from the first to the second layer, the vacancy migrates between 


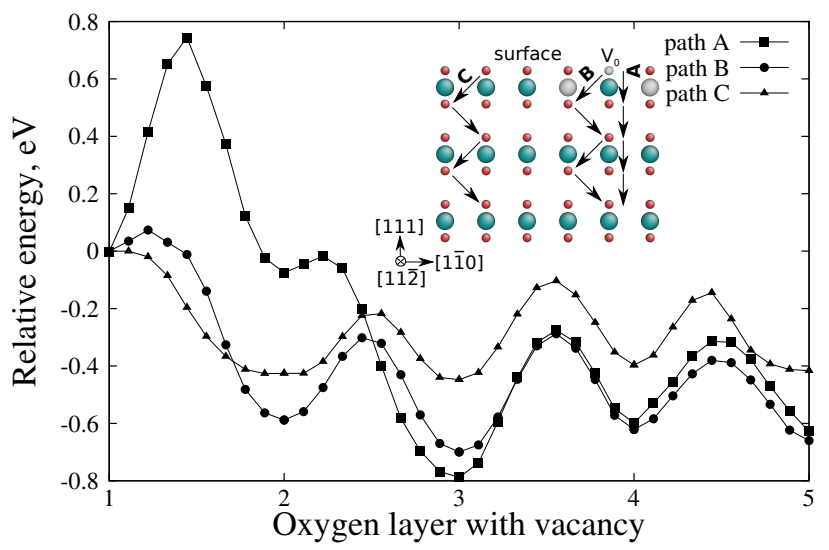

Figure 6: Energy profile for $\mathrm{V}_{\mathrm{o}}$ migration along paths A, B, and C near (111) YSZ surface. Vacancy position is given as a number (counted from the surface) of atomic layer with it. Examined paths of vacancy migration are illustrated in the inset image with a side view of the surface. Zirconium ions are shown by big green circles, yttrium ions - big gray circles, oxide ion - small red circles, initial position of oxygen vacancy in paths A and B - small gray circle. Arrows indicate directions of the oxygen vacancy migration.

the dopants and stays NN to them. The vacancy becomes NNN to the dopants in the third layer for paths $\mathrm{A}$ and $\mathrm{B}$. Along path $\mathrm{B}$, the vacancy in the second layer is NN to one of the dopants and NNN to the other. Path $\mathrm{C}$ is geometrically equivalent to path B but located at some distance from the dopants in a yttrium depleted area.

Energy profile for $\mathrm{V}_{\mathrm{o}}$ migrations along the three paths is shown in Fig. 6 . For all three paths we can see that the total energy of the system is higher when $V_{o}$ is located on the surface. This indicates that the vacancy prefers to be located in the subsurface region than on the surface. This is in a good agreement not only with results of our MD simulations of oxygen diffusion at elevated temperature but also with results reported by Mayernick et al. using the ReaxFF method [48], where the authors observed higher oxygen vacancy concentration in the subsurface oxygen layer compared with the outermost layer of the (111) YSZ surface. Moreover, this result agrees with that of Xia et al. [24] reported in the DFT study of yttrium segregation at the (111) surface. The authors obtained lower defect formation energy of $\mathrm{Y}-\mathrm{V}_{0}-\mathrm{Y}$ defect cluster when its location corresponds to $V_{\text {o }}$ position 2 on path $A$ in our study, compared to position 1. DFT calculations by Cucinotta et al. 


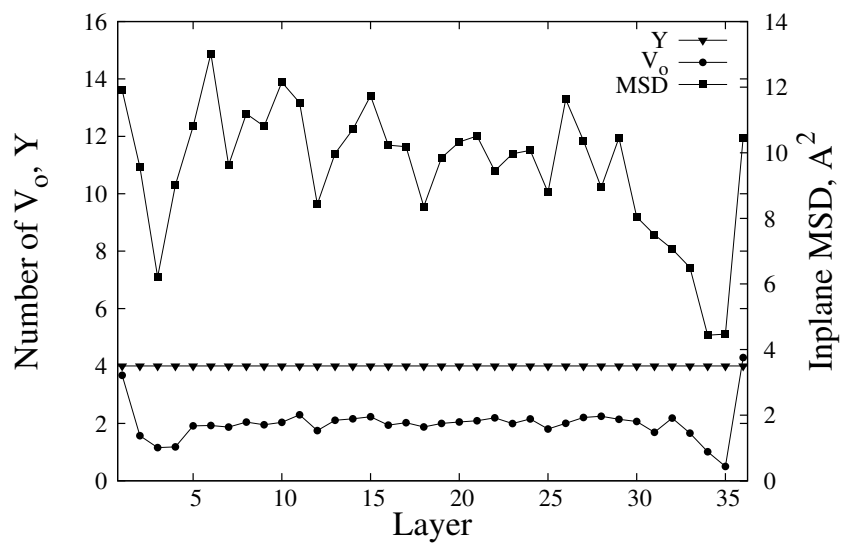

Figure 7: Distribution of yttrium dopants, oxygen vacancies and inplane MSD over atomic layers in $8 \mathrm{~mol} \%$ (110)YSZ slab with evenly distributed Y atoms along the slab's thickness.

also confirm the preference for the vacancies to occupy positions in bulk YSZ rather than at the (111) surface [49].

\subsection{3. (110) surface: $M D$ calculation}

We demonstrated for the (111) surface that there is a correlation between distributions of yttrium dopants and oxygen vacancies along the thickness of YSZ slabs. Thus, since Xia et al. [47] reported no evidence of yttrium segregation at the (110) surface, in this section we present results only for even distribution of yttrium ions. In Fig. 7 we show distribution of oxygen vacancies and in-plane MSD for one of the performed simulations for $8 \mathrm{~mol} \%$ YSZ slab with the size of $21.7 \AA$ and $20.5 \AA$ along the $x$ and $y$ directions, respectively. The thickness of the slab was equal to $61.6 \AA$ containing 36 atomic layers. Average oxygen vacancy distribution over the layers was calculated over a steady-state period of $150 \mathrm{ps}$. The thickness of the imaginary layers was chosen in a way to contain only one atomic layer.

In contrast to the (111) surface, we observe a higher vacancy concentration at the outermost oxygen layers, which is accompanied by depletion in several adjacent subsurface layers. The lack of vacancies in those layers leads to the presence of local minimums on the inplane MSD curve. The averaged numbers of yttrium ions and oxygen vacancies in each layer of the bulk region are equal to the ideal values of 4 and 2, respectively.

Another distinctive effect observed for the (110) surface is oxide ion migra- 
tion within outermost oxygen layer along $\langle 110\rangle$ direction, which corresponds to the migration of oxide ion $\mathrm{B}$ to initial position of $\mathrm{V}_{\mathrm{o}}$ shown in Fig. 8 by the open circle. As a result of this migration, oxide ion $\mathrm{C}$ shifts towards the initial position of anion $\mathrm{B}$ by half of the initial distance between them, while ion $\mathrm{A}$ shifts to its initial position $\mathrm{A}_{0}$ before introducing the oxygen vacancy. In order to understand why the oxide ion migration becomes possible along this path, we performed NEB analysis and compared energy barriers for oxide ion migration along the $\langle 100\rangle$ and $\langle 110\rangle$ directions. These results are presented in the next section.

\subsection{4. (110) surface: NEB analysis}

The two studied migration paths of oxide ion within the outermost layer of the (110) surface and corresponding atomic shifts are illustrated in the inset image in Fig. 8. Path $\mathrm{B}-\mathrm{A}$ is along $\langle 110\rangle$ direction, and path $\mathrm{D}-\mathrm{A}$ is along $\langle 100\rangle$ direction; atomic shifts are shown by dashed and solid lines, respectively. The energy barriers are $0.31 \mathrm{eV}$ and $0.45 \mathrm{eV}$ for the $\langle 100\rangle$ and $\langle 110\rangle$ directions, respectively. These values are more comparable with each other than corresponding barriers in bulk. Indeed, corresponding energy barriers for vacancy migration in bulk zirconia are $0.44 \mathrm{eV}$ and $3.42 \mathrm{eV}$. For $\langle 110\rangle$ direction in bulk, a significantly higher energy barrier equal to $2.5 \mathrm{eV}$ was also estimated by Kaiser-Bischoff et al.[50] compared with 1.07-1.20 eV for $\langle 100\rangle$. However, the authors presumed that the jumps along $\langle 110\rangle$ could be possible at high temperatures or high vacancy concentrations. In our case, it is not only the higher vacancy concentration at the (110) surface, but also the lower energy barrier, that facilitates $\langle 110\rangle$ jumps at the surface.

Tendency of the oxygen vacancy segregation at the (110) surface was supported by the performed NEB analysis of oxygen vacancy migration along the path shown in Fig. 9. The vacancy in the second outermost layer turned out to be unstable and migrated to the outermost layer. This behavior is in agreement with the MD calculations performed at elevated temperature indicating vacancy segregation at the (110) surface. Furthermore, according to Fig. 9, vacancies tend to move to the outermost layer not only from the adjacent atomic layer but also from the deeper bulk regions.

The reason why oxygen vacancies prefer (110) to (111) surface can be qualitatively explained by analysis of atomic coordination numbers. In the outermost layer of the O-terminated (111) surface, an oxide ion is a neighbor to 3 zirconium ions and 3 oxygen ions. In the outermost layer of the (110) surface, an oxide ion is also a neighbor to 3 zirconium ions but to 4 oxygen 


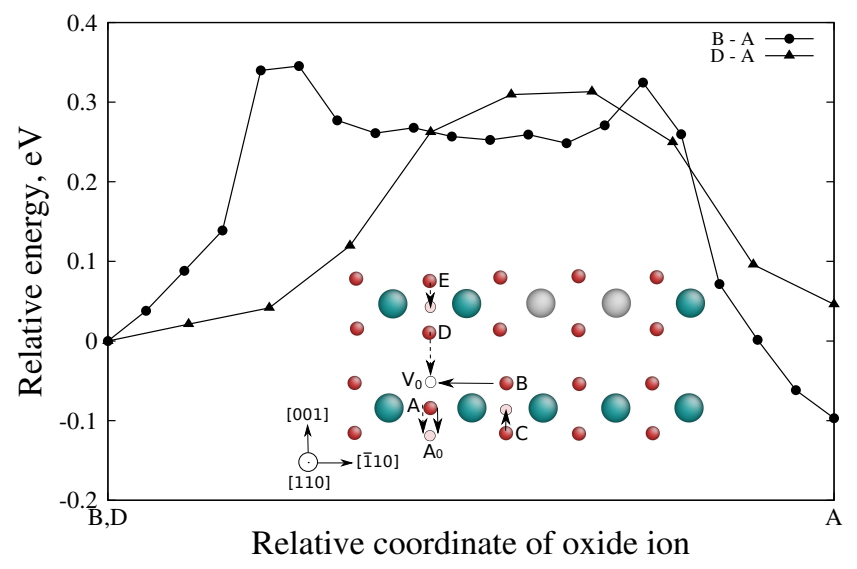

Figure 8: Energy profile for migration of oxide ions along paths B - A and D - A in the outermost layer of the (110) YSZ surface, whose top view is shown in the inset image. Zirconium ions are shown by big green circles, yttrium ions - big gray circles, oxide ions - small red circles, initial position of oxygen vacancy - small gray circle. Small light red circles point final positions of atoms. Solid arrows show rearranging of ions during migration of the oxide ion from B site, dashed arrows - from D site.

ions. Now, if we regard the oxygen vacancy as the consequence of introducing positive neutralizing charge at an oxide ion site on the surfaces, then the preference for the (110) surface can be explained by one extra interaction of the positive charge with an oxide ion. Moreover, the (110) surface of pure cubic zirconia has higher energy than the (111) surface, which is generally believed to be more favorable for the vacancy formation. However, preference for the vacancies to be at the (110) surface compared to the bulk region can not be easily explained; this effect will be confirmed in the next section by DFT approach.

\subsection{5. (110) surface: DFT validation of $M D$ results}

To support our MD results for the (110) surface, we performed two types of DFT calculations. The first one is aimed to estimate diffusion barrier for oxide ion jumps along $\langle 110\rangle$ direction on the surface, the second one - to validate tendency of oxygen vacancy segregation at the surface. Both calculations were based on the same (110) slab model consisting of six atomic layers. Two yttrium dopants were introduced in the outermost layer at the same positions as shown in the inset image of Fig. 8, one oxygen vacancy 


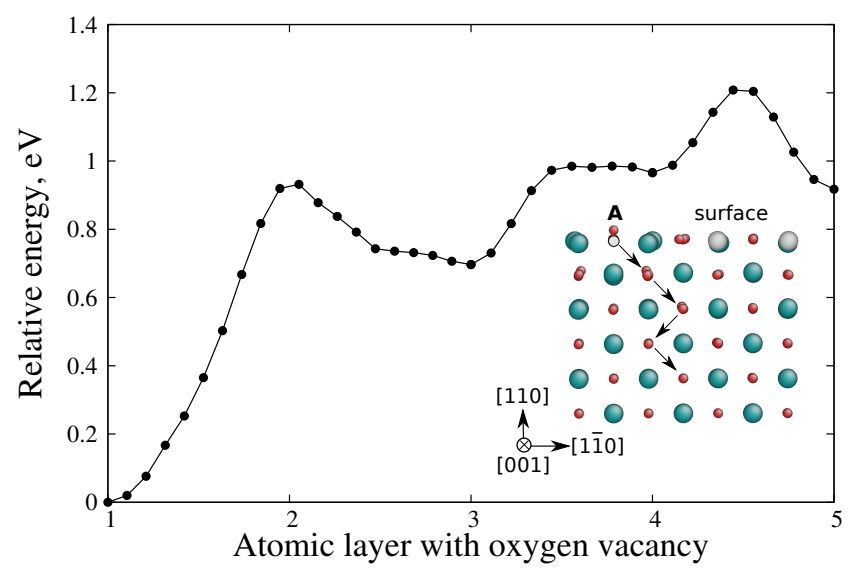

Figure 9: Energy profile of oxygen vacancy migration from (110) YSZ surface into bulk region. The inset image shows side view of the (110) zirconia surface doped by one $\mathrm{Y}_{2} \mathrm{O}_{3}$ unit. Zirconium ions are shown in the inset image by big green circles, yttirum ions - big gray circles, oxygen ions - small red circles, initial position of the oxygen vacancy - small gray circle.

was also introduced to keep stoichiometry. The model contained 107 atoms. Two bottom atomic layers were kept fixed during relaxation.

The energy barrier for oxygen ion migration along $\langle 110\rangle$ direction was estimated as the energy difference between two configurations: the first one is with atom B in at its initial position, the second - with atom B located halfway to the initial position of $\mathrm{V}_{\mathrm{o}}$. The difference is $1.2 \mathrm{eV}$, which is within the range of $0.2-1.4 \mathrm{eV}$ estimated by Pornprasertsuk et al. [12] for $\langle 100\rangle$ direction in bulk. This supports our MD results of a high probability of $\langle 110\rangle$ jumps on the (110) surface. Interestingly, it has been reported that atom A undergoes significant vertical displacement of around $0.7 \AA[26,25]$ during relaxation, which can be seen in the inset image in Fig 9. However, according to the DFT calculation, when atom $B$ moves towards $V_{o}$ site, it rises over the surface by even larger $1.6 \AA$ at the intermediate position. Our interatomic potential predicts very close vertical shift equal to $1.7 \AA$.

Vacancy segregation at the (110) surface observed in MD simulations was also confirmed by DFT calculations. Indeed, energy of the (110) slab with oxygen vacancy initially placed at site B in Fig. 8 is $0.81 \mathrm{eV}$ lower than that with the vacancy in the third atomic layer. This is in a good agreement with the energy profile in Fig. 9, where this energy difference is equal to $0.70 \mathrm{eV}$. 
It is worth noting that the vacancy in the second outermost layer was also unstable during DFT relaxation and migrated to the surface. This gives another credit to our interatomic potential in terms of a good description of diffusion barriers near free surfaces.

It is important to draw attention to the significance of atomic relaxation of the doped (110) surface. The DFT-calculated energy of the unrelaxed (110) slab with oxygen vacancies in the outermost layer is actually higher than that with vacancies in the third atomic layer. The importance of atomic relaxation of the (110) surface with oxygen vacancies was also reported by Nolan et al. [51] in their DFT study of the oxygen vacancy formation energy in ceria. The authors found that the vacancy formation energy derived for the unrelaxed (110) surface was higher than that for the (100) surface. However, the vacancy formation energy obtained from the relaxed (110) surface was found to be smaller than that calculated for the relaxed (100) surface. This indicates that the significant relaxation of the surface structure is responsible for the small vacancy formation energy at the doped (110) surface, while the doped (111) surface undergoes only modest relaxation.

Obtained results for (111) and (110) surfaces may indicate different routes of oxide ion migration to the TPBs formed by these surfaces. Indeed, two considered surfaces possess lowest surface energies compared with other low index surfaces of cubic zirconia, such as (100) and (310) [47], which indicates dominance of the (111) and (110) surfaces. Moreover, these surface orientations can form very stable interfaces and TPB structures with nickel. For example, Matsunaga et al. [52] found the work of separation equal to $0.5 \mathrm{~J} / \mathrm{m}^{2}$ and $4.4 \mathrm{~J} / \mathrm{m}^{2}$ for the $\mathrm{O}$ - and $\mathrm{Zr}$-terminated $\mathrm{Ni}(111) / \mathrm{ZrO}_{2}(111)$ interfaces, respectively. For the $\mathrm{Ni}(100) / \mathrm{ZrO}_{2}(110)$, Muñoz et al. [53] reported the work of separation equal to $0.9 \mathrm{~J} / \mathrm{m}^{2}$. According to our results, at the TPBs formed by (111) YSZ surface, the ions are more likely to move from adjacent subsurface layers. In contrast, there should be a higher probability of the ionic transport towards the TPBs along the (110) surface due to the reduced energy barrier of oxide ion jumps along $\langle 110\rangle$ direction. Moreover, energetic preference of the oxygen vacancies for being located at the (110) surface indicates lower vacancy formation energy at the surface, compared with more stable (111) surface, which, in turn, suggests higher catalytic activity of the (110) surface. 


\section{Conclusion}

We employed our interatomic potential for YSZ to study oxide ion diffusion in bulk YSZ and near two low energy surfaces, namely (111) and (110). Results presented for bulk YSZ demonstrate that our potential can reproduce main properties of oxide ion diffusion: activation energy, conductivity, optimal concentration equal to $8 \mathrm{~mol} \% \mathrm{YSZ}$. As for the oxide ion diffusion near free surfaces, it was confirmed in our previous study [25] that the local atomic environment there can be significantly different from that in bulk due to different atomic coordination and relaxation of the surfaces. The latter is especially pronounced for the (110) surface and, according to the present study, results in facilitated oxide ion diffusion at the surface not only due to the higher oxygen vacancy concentration, but also due to the activation of jumps along $\langle 110\rangle$ direction. We provided support of these two phenomena by corresponding DFT calculations. However, effect of a free surface on oxide ion diffusion depends on the surface orientation. Indeed, in contrast to the (110) surface, there is a tendency of oxygen vacancy drifting from the outermost layer of the (111) surface, which leads to suppressed oxide ion diffusion in that region.

[1] J. Kondoh, T. Kawashima, S. Kikuchi, Y. Tomii, Y. Ito, J. Electrochem. Soc. 145 (1998) 1527.

[2] S. Badwal, J. Mater. Sci. 19 (1984) 1767.

[3] M. S. Khan, M. Islam, D. Bates, J. Mater. Chem. 8 (1998) 2299.

[4] Y. Tang, Q. Zhang, K.-Y. Chan, Chem. Phys. Lett. 385 (2004) 202.

[5] T. P. Perumal, V. Sridhar, K. Murthy, K. Easwarakumar, S. Ramasamy, Physica A: Statistical Mechanics and its Applications 309 (2002) 35.

[6] R. Devanathan, W. Weber, C. Singhal, J. Gale, Solid State Ionics 177 (2006) 1251.

[7] O. J. Durá, M. A. López de la Torre, L. Vázquez, J. Chaboy, R. Boada, A. Rivera-Calzada, J. Santamaria, C. Leon, Phys. Rev. B 81 (2010) 184301.

[8] A. Kushima, B. Yildiz, J. Mater. Chem. 20 (2010) 4809. 
[9] M. Youssef, B. Yildiz, Phys. Rev. B 89 (2014) 024105.

[10] M. Kilo, C. Argirusis, G. Borchardta, R. A. Jackson, Phys. Chem. Chem. Phys. 2219 (2003) 5.

[11] F. Shimojo, T. Okabe, F. Tachibana, M. Kobayashi, H. Okazaki, J. Phys. Soc. of Japan 61 (1992) 2848.

[12] R. Pornprasertsuk, P. Ramanarayanan, C. B. Musgrave, F. B. Prinz, J. Appl. Phys. 98 (2005) 103513.

[13] R. Krishnamurthy, Y.-G. Yoon, D. J. Srolovitz, R. Car, J. Am. Ceram. Soc. 87 (2004) 1821.

[14] H. Okazaki, H. Suzuki, K. Ihata, Journal of the Physical Society of Japan 63 (1994) 3556.

[15] H. Okazaki, H. Suzuki, K. Ihata, Physics Letters A 188 (1994) 291.

[16] E. Lee, F. B. Prinz, W. Cai, Phys. Rev. B 83 (2011) 052301.

[17] K. Suzuki, M. Kubo, Y. Oumi, R. Miura, H. Takaba, A. Fahmi, A. Chatterjee, K. Teraishi, A. Miyamoto, Applied Physics Letters 73 (1998) 1502 .

[18] E. Fabbri, D. Pergolesi, E. Traversa, Sci. Texhnol. Adv. Mater. 11 (2010) 054503 .

[19] N. Schichtel, C. Korte, D. Hesse, J. Janek, Phys. Chem. Chem. Phys. 11 (2009) 3043.

[20] C. Korte, N. Schichtel, D. Hesse, J. Janek, Monatsh. Chem. 140 (2009) 1069.

[21] B. J. Morgan, P. A. Madden, Phys. Rev. B 89 (2014) 054304.

[22] C. Korte, A. Peters, J. Janek, D. Hesse, N. Zacharov, Phys. Chem. Chem. Phys. 10 (2008) 4623.

[23] M. Alfredsson, C. R. A. Catlow, Chem. Phys. Phys. Chem. 3 (2001) 4129.

[24] X. Xia, R. Oldman, R. Catlow, J. Mater. Chem. 21 (2011) 14549. 
[25] A. Iskandarov, A. Kubo, Y. Umeno, J. Phys.: Condens. Matter 25 (2015) 015005.

[26] G. Ballabio, M. Bernasconi, F. Pietrucci, S. Serra, Phys. Rev. B. 70 (2004) 075417.

[27] M. Vogler, A. Bieberie-Hütter, L. Gauckler, J. Warnatz, W. Bessler, J. Electrochem. Soc. 156 (2009) B663.

[28] S. C. Ammal, A. Heyden, J. Phys. Chem. Lett. 3 (2012) 2767.

[29] P. Tangney, S. Scandolo, J. Chem. Phys. 117 (2002) 8898.

[30] A. J. Rowley, P. Jemmer, M. Wilson, P. A. Madden, J. Chem. Phys. 108 (1998) 10209.

[31] D. Wolf, P. Keblinski, S. R. Phillpot, J. Eggebrecht, J. Chem. Phys. 110 (1999) 8254.

[32] P. Brommer, P. Beck, A. Chatzopoulos, F. Gähler, J. Roth, H.-R. Trebin, J. Chem. Phys. 132 (2010) 194109.

[33] P. Beck, P. Brommer, J. Roth, H.-R. Trebin, J. Chem. Phys. 135 (2011) 234512.

[34] M. Parinello, A. Rahman, J. Appl. Phys. 52 (1981) 7182.

[35] G. Kresse, J. Haffner, Phys. Rev. B. 47 (1993) 558.

[36] G. Kresse, J. Furthmüller, Phys. Rev. B. 54 (1996) 11169.

[37] G. Kresse, D. Joubert, Phys. Rev. B. 59 (1999) 1758.

[38] X. Xia, Computational modelling study of yttria-stabilized zirconia, Ph.D. thesis, University College London (2010).

[39] A. Nakamura, J. B. W. Jr., J. Electrochem. Soc. 133 (1986) 1542.

[40] C. C. T. Yang, W. C. J. Wei, A. Roosen, Mater. Chem. Phys. 81 (2003) 134.

[41] S. Hull, Rep. Prog. Phys. 67 (2004) 1233. 
[42] K. C. Lau, B. I. Dunlap, J. Phys.: Condens. Matter 23 (2011) 035401.

[43] P. Lindan, M. Gillan, J. Phys.: Condens. Matter 5 (1993) 1019.

[44] P. S. Manning, J. D. Sirman, R. A. D. Souza, J. A. Kilner, Solid State Ionics 100 (1997) 1.

[45] P. S. Manning, J. D. Sirman, J. A. Kilner, Solid State Ionics 93 (1997) 125.

[46] H. Solmon, C. Monty, M. Filal, G. Petot-Ervas, C. Petot, Solid State Phenomena 41 (1995) 103.

[47] X. Xia, R. Oldman, R. Catlow, Chem. Mater. 21 (2009) 3576.

[48] A. D. Mayernick, M. Batzill, A. C. T. van Duin, M. J. Janik, Surf. Sci. $604(2010) 1438$.

[49] C. S. Cucinotta, M. Bernasconi, M. Parrinello, Phys. Rev. Lett. 107 (2011) 206103.

[50] I. Kaiser-Bischoff, H. Boysen, C. Scherf, T. Hansen, Phys. Chem. Chem. Phys. 7 (2005) 2061.

[51] M. Nolan, S. C. Parker, G. W. Watson, Surface Science 595 (2005) 223.

[52] K. Matsunaga, T. Sasaki, N. Shibata, T. Mizoguchi, T. Yamamoto, Y. Ikuhara, Physical Review B 74 (2006) 125423.

[53] M.C.Muñoz, S. Gallego, J. Beltrán, J. Cerdá, Surface Science Reports 61 (2006) 303. 